\title{
ARC DISCHARGE SLIDING OVER A CONDUCTING SURFACE
}

\author{
F. A. van Goor, ${ }^{1}$ S. V. Mit'ko, ${ }^{2}$ V. N. Ochkin, ${ }^{2}$ A. P. Paramonov, ${ }^{2}$ and W. J. Witteman ${ }^{1}$
}

${ }^{1}$ University of Twente, Department of Applied Physics, P.O. Box 217, 7500 AE Enschede, The Netherlands

${ }^{2}$ P. N. Lebedev Physical Institute, Russian Academy of Sciences, Leninsky Pr. 53, Moscow 117924, Russia

\begin{abstract}
Results of experimental and theoretical studies of the arc discharge which slides over the surface of a conductor are reported. Experiments were performed in air and argon ambients at various pressures. It is found that the velocity of the discharge plasma front depends linearly on the strength of the average electric field ahead of the front. A physical model of this phenomenon for the range of electric fields near the discharge threshold was developed. It is demonstrated that the mechanism related to thermal conductivity is responsible for the plasma front propagation.
\end{abstract}

\section{Introduction}

Most likely, the phenomenon of discharge-plasma propagation over the surface of a conductor was first observed experimentally in 1967 [1]. The experiment [1] was related to the studies of surface breakdown in the insulators of power-transmission lines under high-humidity and heavy-contamination conditions. In [1], the discharge over the surface of a water jet coming from a faucet was studied. The conductivity of water was varied by adding a $12 \%$ salt solution. The faucet served as a ground electrode, whereas a thin metal wire placed near the water surface was used as a high-voltage electrude. A voltage if up to $11 \mathrm{kV}$ was fed to the wire from a dc power supply.

As the voltage was applied, the initiation of an arc discharge was observed; the discharge channel moved over the jet surface in the direction of the ground electrode. The maximum velocity of the motion measured at a voltage of $11 \mathrm{kV}$ was $600 \mathrm{~m} / \mathrm{s}$. The measurements of the velocity of the discharge front as a function of the conductivity of water demonstrated that, for each value of conductivity, there is a corresponding threshold current below which a stationary arc discharge is observed between the high-voltage electrode and the nearest point of the water surface. With increase in conductivity, the threshold current tended to increase. However, data about the polarity of the applied voltage, length and diameter of the jet, and influence of the jet characteristics on the observed velocity of plasma propagation were not reported in [1].

Later on, a new form of discharge was studied [2]. The possibility of generating a low-temperature plasma at the gas-aqueous electrolyte interface by applying voltage pulses of microsecond duration was considered [2]; the efficiency of implementing such discharges for purification of aqueous media from toxic and poorly removable compounds (such as benzapyrene and phenol) and also from microflora including pathogenic bacteria was examined as well. Power to the discharge was supplied from a capacitor bank with capacitance from 0.1 to $3 \mu \mathrm{F}$ with a maximum charging voltage of $45 \mathrm{kV}$. The electrolyte was put in a container $160 \mathrm{~cm}$ in diameter. The conductivity of the electrolyte was varied within the range of $10^{-6}-10^{-1} \Omega^{-1} \mathrm{~cm}^{-1}$. Various geometries of a discharge gap were used with the gap width varying from 5 to $80 \mathrm{~cm}$ : tip-tip, tip-flat surface, and tip-cylindrical surface.

With the voltage applied, two plasma channels appeared and traveled toward each other from the electrodes of opposite polarity with a velocity between $5 \cdot 10^{3} \mathrm{~cm} / \mathrm{s}$ and $10^{7} \mathrm{~cm} / \mathrm{s}$. It was noted that there was a significant difference between the velocities of leaders of opposite polarity in the case of low conductivity of

Translated from a manuscript submitted October 14, 1996. 
the electrolyte. Thus, with the conductivity decreased to $10^{-6} \Omega^{-1} \mathrm{~cm}^{-1}$, the velocity of the anode-directed leader is several orders of magnitude higher than that of the cathode-directed leader. With the conductivity being higher, the leaders of opposite polarities traveled with the same velocity.

The mechanism of breakdown was related to the transfer of the electrode potential to the leader's edge as a result of high conductance of the plasma channel and to the emergence of the region of the high-strength electric field ahead of the plasma front. Because of this, discharge initiation occurs at low voltages between the electrodes in the case of wide gaps.

A search for methods of initiating extended discharges with the use of indestructible components resulted in the fact that the phenomenon of sliding discharge was observed again in [3]. The experiments were performed in the air ambient at atmospheric pressure. A carbon-graphite rod with a diameter of $2 \mathrm{~mm}$, length of $10.5 \mathrm{~mm}$, and conductivity of $30 \Omega^{-1} \mathrm{~cm}^{-1}$ was used for initiation of the discharge. The charging voltage for a storage capacitor of several-millifarad capacitance was varied from 1 to $3 \mathrm{kV}$. The spatialtemporal pattern of breakdown was examined by the schlieren photography of the discharge gap.

The emergence of two arc channels, which moved from both electrodes to the center of the discharge gap and had the same velocities, was observed when the applied voltage exceeded $15 \mathrm{kV}$. The maximum velocity, equal to $1.5 \cdot 10^{4} \mathrm{~cm} / \mathrm{s}$, was attained at a voltage of $3 \mathrm{kV}$. Even with such a low velocity of advance, the shock wave was in evidence ahead of the arc-channel front. It was noted that the propagation of the arc channel could be stopped by covering a portion of the carbon-graphite rod with an insulating disk.

The propagation of the sliding arc discharge was qualitatively attributed to the heating of gas ahead of the plasma front due to thermal conductivity. The front velocity estimated in [3] on the basis of this model coincided with the experimentally measured one. However, the model involved crude approximations, and also the data on the dependence of the front propagation on applied voltage, current, and the type and pressure of ambient gas were lacking; all this prevented the authors of [3] from subjecting their hypothesis to a more detailed testing.

Although the main objective of [3] was to develop a system for the initiation of pulse-recurrent behavior, the carbon-graphite rod withstood 3-4 discharge pulses without destruction, with the energy supplied being about several kilojoules. With lowered energy-supply levels, the rod lifetime was substantially longer; however, the energy required to initiate the discharge was relatively much higher. Apparently because of this, the studies of this method were abandoned.

The method of initiation under consideration was further developed in [4-7]. It was suggested that a NiMn ferrite, which has an appreciable conductivity in the normal state, be used [4] as an initiating component. It was demonstrated that in the final stage of initiation of a high-current pulsed arc, the emission characteristics of the discharge with a length of $24 \mathrm{~cm}$ compare well with those of the discharge initiated with an exploding wire [5]. The emission and gas-dynamics characteristics of discharge in the stage of initiation of a highcurrent pulsed arc were studied in [6]; there, a numerical algorithm was developed to solve the two-dimensional problem on dynamics of surface discharge with allowance for the energy transport by radiation, and the results of calculations were found to agree satisfactorily with experimental data. In [7], an initiating component of $90 \mathrm{~cm}$ in length was used in the design of a high-power radiation source for optical pumping of excimer lasers. The long-term $\left(10^{4}-10^{5}\right.$ pulses $)$ pulse-recurrent mode of arc initiation with energy supply of about $\sim 10^{4} \mathrm{~kJ}$ and tolerable erosion of the surface of the initiating component were demonstrated.

Thus, attractive features of the mode of discharge discussed above include a low initiation voltage, the possibility of influencing the discharge characteristics over a wide range, and a wide choice of conductors in both liquid and solid states. However, in contrast to the closest analogue (i.e., a discharge sliding over the surface of an insulator), the fundamental issues of the physics of discharge formation over a conductor remain practically open. Thus, in the above-mentioned publications, one can find two radically different points of view, which relate the mechanism of discharge propagation either to the ionization of cold gas in the enhanced field ahead of the plasma front or to the processes of thermal conduction. Both hypotheses 


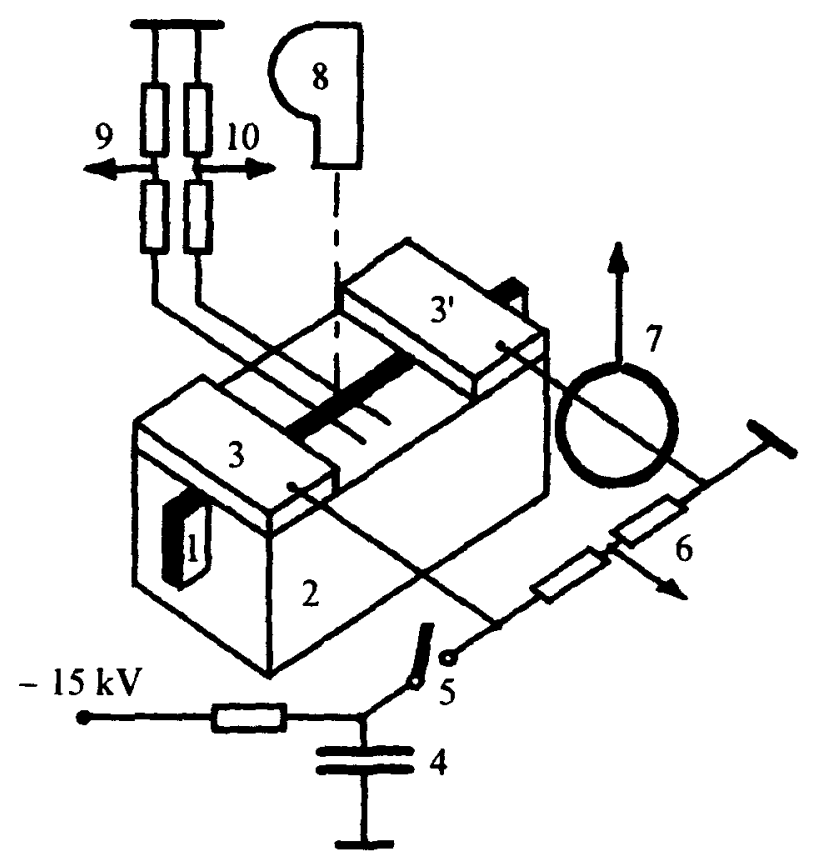

Fig. 1. Schematic of experimental setup: 1) plate of an M700NM-brand NiMn ferrite; 2) tetrafluoroethylene holder; $3,3^{\prime}$ ) electrodes; 4) storage capacitor; 5) electrical-mechanical relay; 6) resistive voltage divider; 7) Rogowski coil; 8) VFU-1 photography system; 9,10 ) electrical probes.

have not been tested experimentally. There is no systematic data available about the dependence of the velocity of the plasma front on the parameters of the discharge circuit, the substrate conductivity, and the composition and pressure of the gas surrounding the conductor. Because of this, the outlook for the practical implementation of this type of discharge remains uncertain. In order to make the above-stated issues more clear, we undertook an experimental and theoretical study of the discharge in question.

\section{Experimental Setup}

Since the systems with a solid conductor are of the most practical interest for initiation of extended plasma formation, a sample of NiMn ferrite that demonstrated good characteristics in laser experiments [7] was used in our experiments with discharge initiation.

The schematic diagram of the experimental setup is shown in Fig. 1. A ferrite plate 1 having a cross section of $1 \times 0.1 \mathrm{~cm}^{2}$ and electrical conductivity of $\sim 0.5 \Omega^{-1} \mathrm{~cm}^{-1}$ was used with an electric-field strength of $\sim 1.5 \mathrm{kV} / \mathrm{cm}$. In order to prevent the plasma channel from branching and bending, we embedded the plate into a tetrafluoroethylene holder 2 . Two copper platelets 3 and $3^{\prime}$, serving as electrodes, were pressed to the narrow face of the ferrite sample and were spaced $7.5 \mathrm{~cm}$ apart. A storage capacitor 4 with charging voltage of up to $15 \mathrm{kV}$ and capacitance varied from 0.1 to $1 \mu \mathrm{F}$ was discharged into the initiation unit with the use of an electrical-mechanical relay 5 . The sample to be studied was placed in a vacuum chamber, which made it possible to perform measurements with the sample in the ambiance of different gases.

We measured the voltage at the electrodes with the use of a resistive divider 6 ; in order to measure the total current through the sample, we employed a Rogowski coil 7 combined with an integrating circuit. The dynamics of the plasma channel was studied with a high-speed photography system 8 , which operated in the slit-sweep mode with time resolution of $0.5 \mu \mathrm{s}$. The electric-field distribution along the plasma column was 


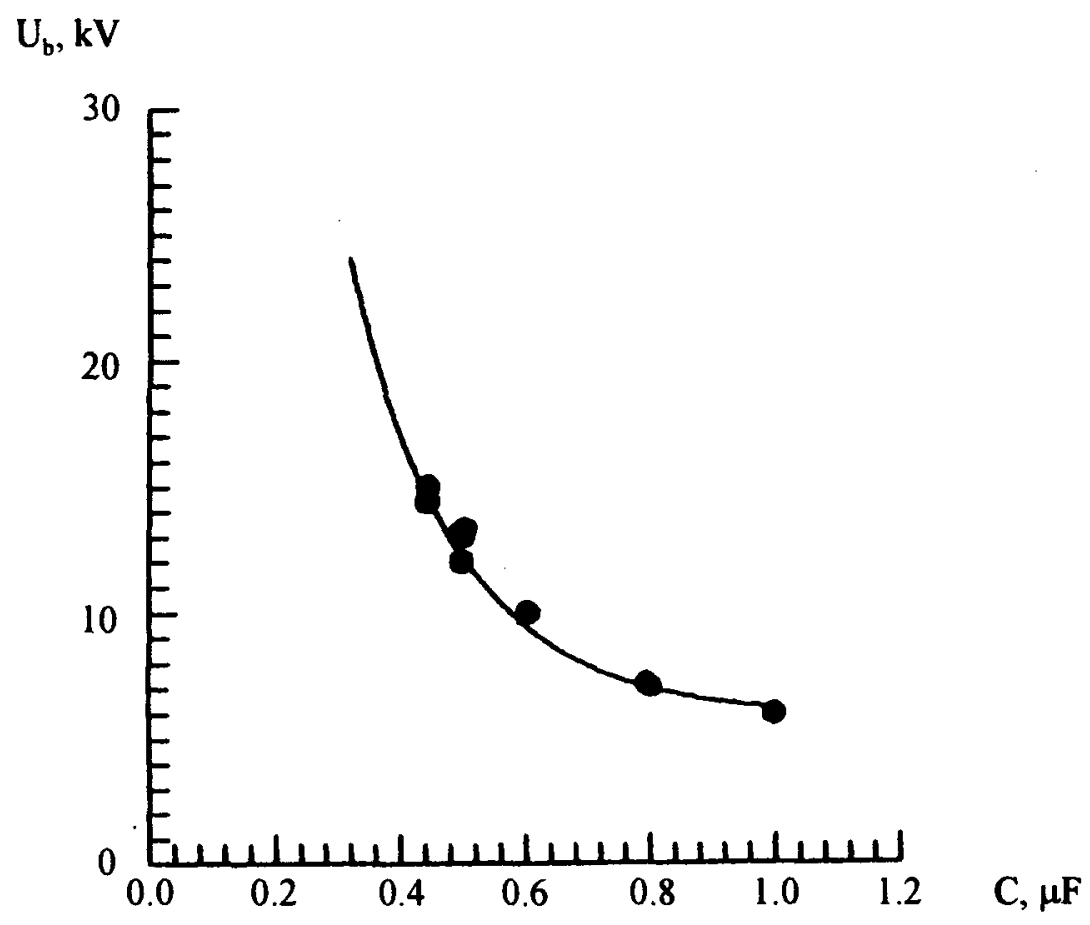

Fig. 2. The voltage $U_{b}$ corresponding to overlap for a discharge in the ambient air at atmospheric pressure as a function of capacitance $C$ of the storage capacitor. The solid line represents the results of calculation with formula (7), and the points are experimental.

studied with the use of probes 9 and 10, which were made from molybdenum wires $0.1 \mathrm{~mm}$ in diameter and were spaced $5 \mathrm{~mm}$ apart. The wires were pressed against the ferrite surface in the middle of the discharge gap.

\section{Experimental Results}

In agreement with $[1,3]$, we found that the process of formation of the propagating plasma leader has a threshold in voltage. Moreover, the threshold voltage appeared to depend on the type and pressure of the gas in which the discharge developed. Thus, a surface discharge in the ambient air under atmospheric pressure cannot be initiated if the initial voltage across the capacitor is lower than $6 \mathrm{kV}$. Furthermore, in this case, short arc channels are observed near the contacts of electrodes with the ferrite surface, and slow discharge of the capacitor occurs through the bulk of the conductor. With the voltage exceeding threshold, we observed the formation of plasma channels which moved from the electrodes toward each other. In our experiments, the development of an anode-directed plasma channel was invariably predominant (irrespective of the polarity of the high-voltage electrode and also of the gas type and pressure). The velocity of the cathode-directed leader was lower by several orders of magnitude.

The further evolution of plasma depends on the magnitude of applied voltage and the capacitance of the storage capacitor. If both the voltage and capacitance were low, we observed an uncompleted type of discharge when the plasma-formation front stops within the discharge gap, and the plasma decays with decreasing current and voltage. With the voltage and capacitance sufficiently large, the plasma leader overlaps the entire discharge gap and a transition to the final stage is observed, i.e., to the initiation of pulsed arc discharge. The dependence of the overlap (breakdown) voltage $U_{b}$ on the capacitance $C$ of the storage 

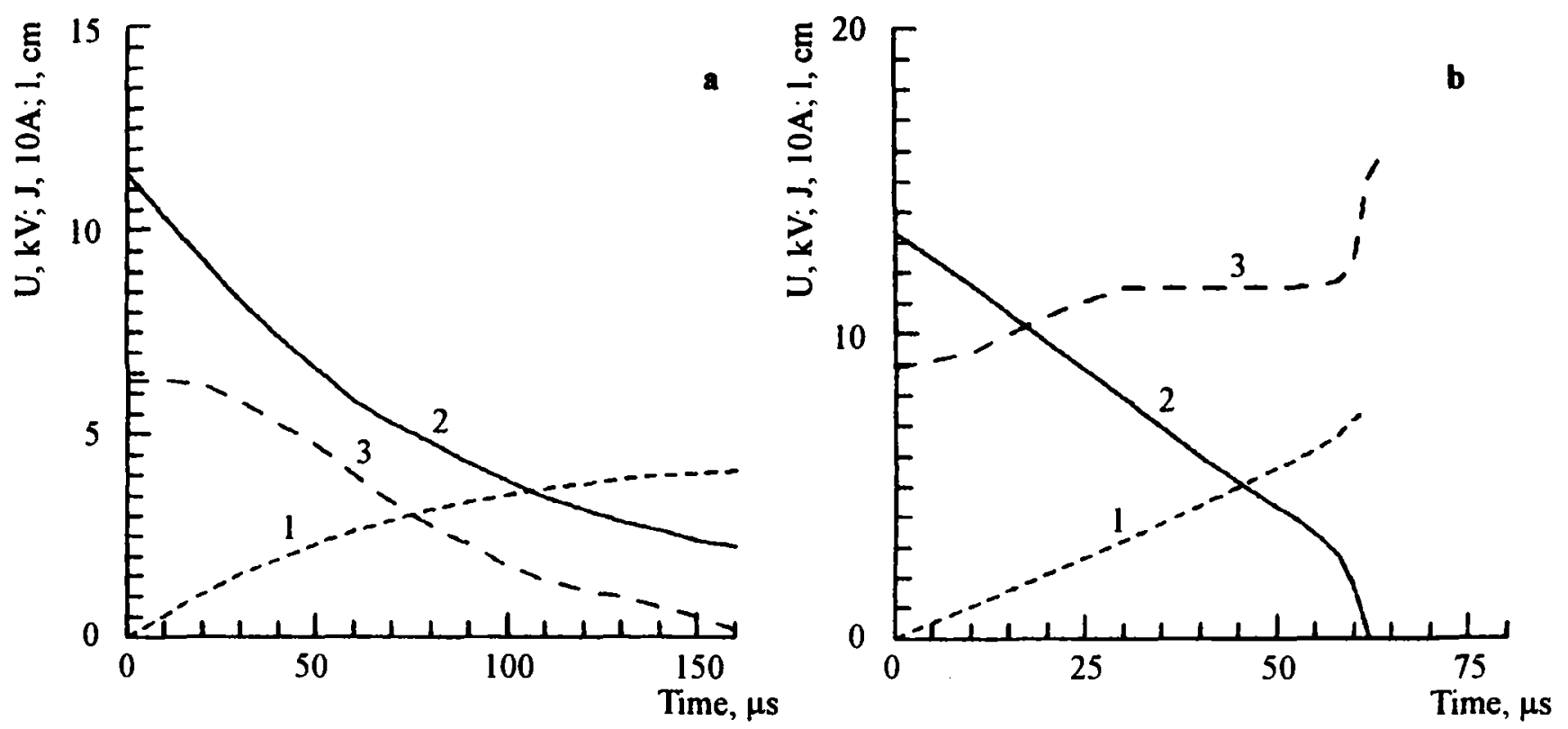

Fig. 3. The parameters of a sliding arc discharge in the ambient air at atmospheric pressure for incomplete [(a) $U=10.4 \mathrm{kV}]$ and complete [(b) $U=13.2 \mathrm{kV}$ ] forms of the discharge: 1) channel length $l ; 2$ ) voltage $U ; 3)$ current $J$. The capacitance was $0.5 \mu \mathrm{F}$.

capacitor is shown in Fig. 2. We measured the lowest initial voltage which still ensured overlapping of the interelectrode gap by the plasma leader of the discharge in the air ambient under atmospheric pressure. It is evident that a slight variation of capacitance in the vicinity of $0.4 \mu \mathrm{F}$ strongly affects the magnitude of $U_{b}$. With increasing capacitance $C$, the voltage $U_{b}$ decreases and tends to a constant value; for $C=1 \mu \mathrm{F}$, $U_{b}=6 \mathrm{kV}$.

Figure 3a and $\mathrm{b}$ shows the representative oscillograms of voltages at electrodes and total current in the discharge circuit, and the time dependence of the length of the anode-directed plasma channel; the lat ter curve was plotted on the basis of streak-camera pictures. The data correspond to the discharge in the air ambient at atmospheric pressure with the capacitance of the storage capacitor being $0.5 \mu \mathrm{F}$. Figure 3a corresponds to an incomplete form of the discharge. As is evident, the formation of plasma occurs against the backgound of a steady decrease of current and voltage and of the corresponding decrease in the velocity of the plasma front. In the case of Fig. 3b, the initial voltage only slightly exceeds the voltage required for the plasma leader to overlap the discharge gap. As can be seen, notwithstanding the large variations in current and voltage in the process of discharge development, the velocity of the leader is found to remain practically constant until the moment of overlap.

It is the latter case of plasma-front propagation with constant velocity that we chose in our experiment, intended to refine the data on the conductivity of the plasma column and to study the electric-field distribution along this column. With this in mind, we measured the time dependence of the potential difference between the high-voltage electrode and each of the electrical probes 9 and 10 (see Fig. 1) arranged at the surface of a ferrite slab. The measurements were performed under the conditions corresponding to Fig. $3 \mathrm{~b}$. The solid curve in Fig. 4 represents the time dependence of the potential difference $\Delta V$ between the cathode and the nearest probe 9 (spaced $3.25 \mathrm{~cm}$ from the cathode). It can be seen that the potential difference $\Delta V$ becomes negligibly small within $28 \mu$ s after the voltage is supplied. This is in complete agreement with the pattern depicted in Fig. 3b, whence it is evident that the plasma front reaches the probe by about $30 \mu \mathrm{s}$. A small 


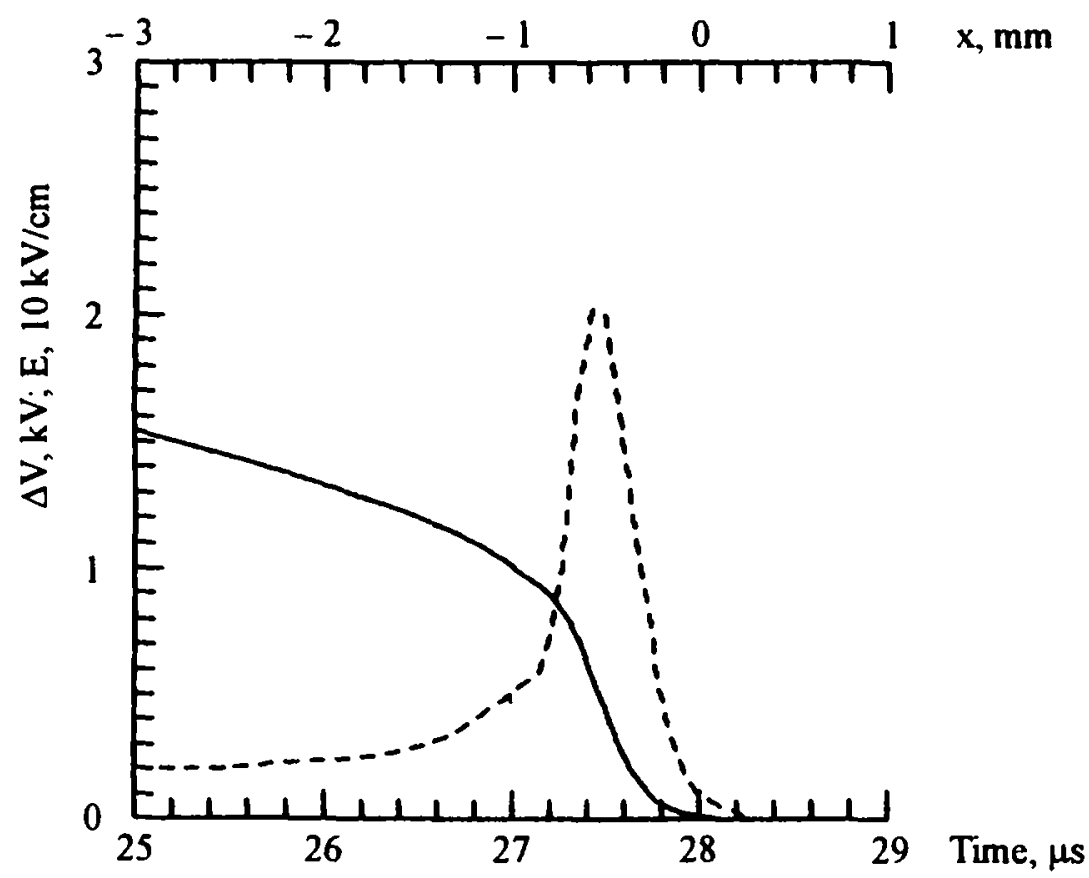

Fig. 4. Spatial distribution of the electric-field strength $E$ ahead of the plasma front. The dashed line corresponds to the electric field $E$ and the solid line illustrates the behavior of the potential difference $\Delta V$ between the anode and cathode.

magnitude of potential difference across the plasma column at a current of about $100 \mathrm{~A}$ indicates that the conductivity of the plasma is appreciably larger than the conductivity of the conductor $\left(0.5 \Omega^{-1} \mathrm{~cm}^{-1}\right)$ and that the plasma temperature is high. Such a dependence featuring a time shift of $5 \mu$ s was obtained for the probe 10 spaced $3.75 \mathrm{~cm}$ from the cathode.

The time delay between signals from the probes separated by a known distance was used to determine the velocity $u$ of the plasma-front motion at a given moment. The velocity determined with the probes was $10^{5} \mathrm{~cm} / \mathrm{s}$. The velocity determined by photography under similar conditions amounted to $1.1 \cdot 10^{5} \mathrm{~cm} / \mathrm{s}$, which closely agrees with the data obtained on the basis of probes.

By making use of the experimentally established fact that the velocity of the plasma front is constant under the above-specified conditions, we were able to plot the spatial distribution of the tangential component of the electric field ahead of the plasma front. The electric field $E(x)$ was calculated from experimental data with the use of the relationship

$$
E(x)=-\frac{1}{u} \frac{d \Delta V(t)}{d t}
$$

Here, $u=10^{5} \mathrm{~cm} / \mathrm{s}$ is the plasma-front velocity, $t$ is time, and $x=u\left(t_{c}-t\right)$ is the coordinate measured from the plasma front, with the moment $t_{c}=28 \mu \mathrm{s}$ corresponding to the traversal of the plasma front through the probe site.

The dependence $E(x)$ (the upper scale) is shown in Fig. 4 by the dashed curve. As is evident, there is an enhanced electric field with maximum strength of about $20 \mathrm{kV} / \mathrm{cm}$ in the region extended by $\sim 0.05 \mathrm{~cm}$ ahead of the plasma front. The mean strength of the electric field $E_{a}$ determined as the ratio of the voltage at electrodes to the distance between the anode and plasma front amounts to about $2 \mathrm{kV} / \mathrm{cm}$ under the above-specified conditions. Thus, the fact that the electric field is appreciably enhanced ahead of the plasma front may be considered as experimentally established. The origin of this enhancement is the transfer of the 


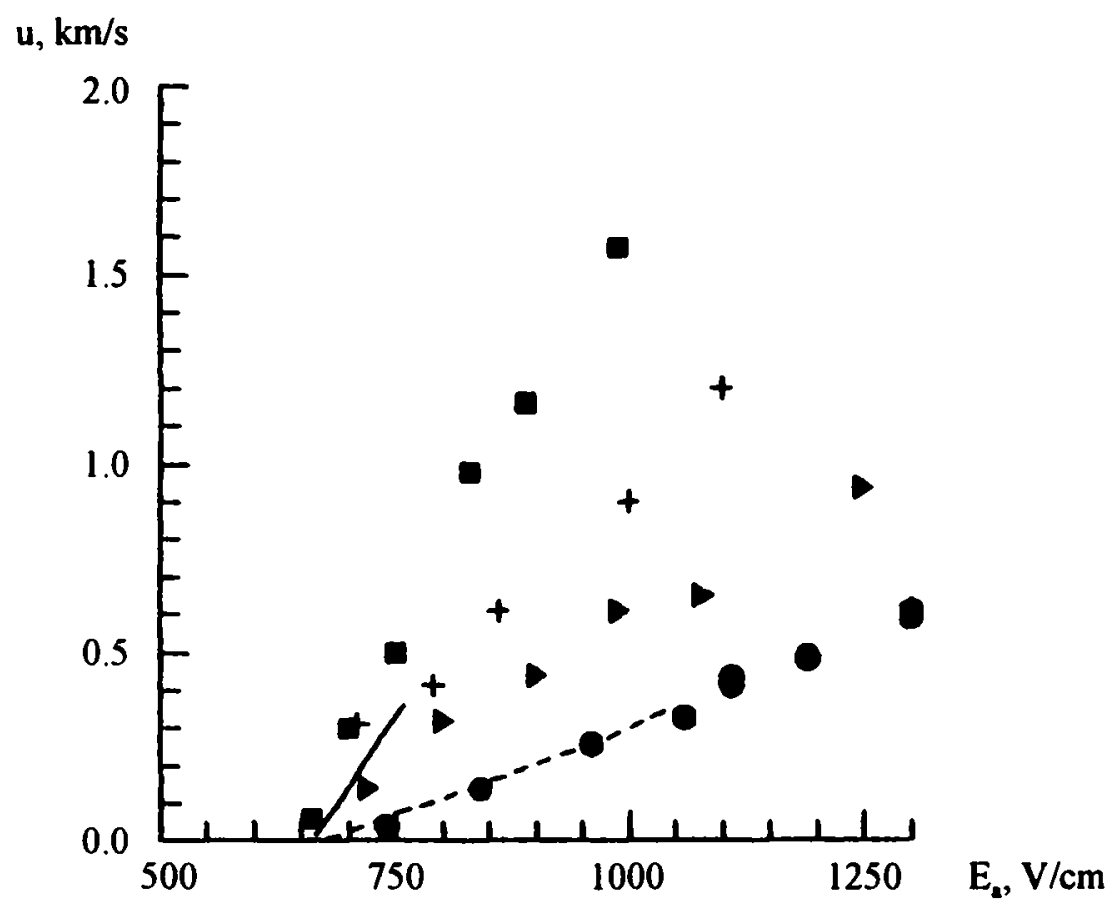

Fig. 5. The velocity $u$ of the anode-directed leader as a function of average field strength $E_{a}$. The results of calculations (see Sec. 4.3) are presented for argon at 1 atm (solid curve) and air at 1 atm (dashed curve). Experimental points correspond to air at $1 \mathrm{~atm}(\bullet)$, air at $0.5 \mathrm{~atm}(\triangleright)$, air at $0.25 \mathrm{~atm}(+)$, and argon at $1 \mathrm{~atm}(\square)$.

cathode potential to the leading edge of the front due to the high conductivity of the latter.

Establishment of the fact that the plasma channel has high conductivity allowed us to deduce the dependence of the leader-propagation velocity on the mean electric field ahead of the plasma front. Figure 5 shows the velocity of the front of the anode-directed leader as a function of the average field strength $E_{a}$ for several pressures of ambient air. A similar dependence for the case of filling the chamber with argon is also shown. As the pressure of air is lowered from 1 atm to $0.25 \mathrm{~atm}$, the threshold for discharge initiation decreases from about $700 \mathrm{~V} / \mathrm{cm}$ to $500 \mathrm{~V} / \mathrm{cm}$ and the curve $u(E)$ steepens.

It was found that experimental data on the velocity of plasma-front propagation may be approximated to a sufficient accuracy by the following relationship:

$$
u=k\left(E_{a}-E_{c}\right)
$$

Here, $u$ is the leader-propagation velocity; $E_{a}$ and $E_{c}$ are the instantaneous and threshold strengths of the average electric field; and $k$ is a constant defining the rate of velocity increase, with $k$ and $E_{c}$ depending on the type and pressure of ambient gas. The experimentally established dependence of the plasma-propagation velocity on the type and pressure of ambient gas indicates that the possible evaporation and desorption of gases from the ferrite surface plays a minor role under the experimental conditions of this study.

\section{Discussion of Results}

\subsection{Criterion for Overlapping of the Discharge Gap}

The questions on the conditions for overlapping of the entire discharge gap by the leader and the delay time of initiation of the high-current phase of an arc discharge with respect to applied voltage pulse are of 
prime practical interest in relation to initiation of an extended arc discharge.

In order to solve these questions, we consider the discharge circuit consisting of a storage capacitor $C$ and a conductor which has resistance per unit length $r_{0} \Omega / \mathrm{cm}$ and length $L$. In accordance with experimental data, we assume that the conductor is completely shunted by the discharge plasma over the entire distance traveled by the plasma front. Moreover, we approximate experimental data for the plasma-front velocity $u$ by expression (2). The system of equations for voltage $U(t)$ across the capacitor and for the distance $l(t)$ between the plasma front and the anode has the form

$$
\begin{aligned}
\frac{d U(t)}{d t} & =-\frac{U(t)}{r_{0} C l(t)} \\
\frac{d l(t)}{d t} & =-u(t) \\
u(t) & =k\left(\frac{U(t)}{l(t)}-E_{c}\right)
\end{aligned}
$$

with initial conditions set as $l(0)=L$ and $U(0)=U_{0}$.

Let us determine the lowest voltage $U_{b}$ which corresponds to the onset of overlap of the discharge gap by the leader. If the initial voltage $U_{0}$ is lower than the voltage $U_{c}=E_{c} L$ required for initiation of the leader, the storage capacitor slowly discharges via the conductor bulk without formation of a surface plasma. If $U_{c}<U_{0}<U_{b}$, an incomplete form of discharge is observed, with the leader propagating with steadily decreasing velocity and ceasing to move within the discharge gap. By contrast, if the initial voltage $U_{0}$ exceeds the breakdown (overlap) voltage $U_{b}$, the leader moves with steadily increasing velocity.

Thus, in the case of $U_{0}=U_{b}$, the plasma-front velocity must be constant; moreover, the capacitor becomes completely discharged by the moment the leader reaches the anode. From the constancy of the propagation velocity $u$, it also follows that the average strength of the electric field $U(t) / l(t)=U_{b} / L$ ahead of the plasma front is constant. In view of this, we can easily derive a solution to the system of equations (3) for the voltage across the capacitor and for the distance between the leader edge and the anode as

$$
\begin{aligned}
U(t) & =U_{b}\left(1-\frac{t}{r_{0} C L}\right), \\
l(t) & =L-k\left(\frac{U_{b}}{L}-E_{c}\right) t .
\end{aligned}
$$

The overlap of the discharge gap occurs at the moment

$$
t_{b}=r_{0} C L,
$$

as follows from the condition $U\left(t_{b}\right)=0$. Thus, the time it takes for the discharge gap to be overlapped with the threshold voltage applied equals the discharge-circuit time constant at the initial instant of time. Substituting (6) into (5) and taking into account that $l\left(t_{b}\right)=0$, we arrive at the following condition for overlap:

$$
F\left(\frac{E_{b}}{E_{c}}-1\right)=1
$$

Here, $F=k r_{0} C E_{c}$ and $E_{b}=U_{b} / L$.

The dependence of the overlap voltage on the capacitance of the storage capacitor was calculated from relationship (7) and is shown by the solid curve in Fig. 2. The calculation was performed for the case of the discharge in air ambient under atmospheric pressure. We used the values of $k=100.8 \mathrm{~cm} / \mathrm{s}$ and $E_{c}=714.5 \mathrm{~V} / \mathrm{cm}$ obtained by approximating the experimental data in Fig. 5 with the use of relationship 
(2). It is evident that an increase in capacitance $C$ results in a decrease in voltage $U_{b}$. There is a satisfactory agreement between the theoretical curve and experimental results.

As follows from (7), there is a minimum overlap voltage $U_{\min }=E_{c} L$ that corresponds to the infinitely large capacitance of the capacitor. Under the conditions of our experiment, this limiting mode corresponds to $U_{\min }=5.4 \mathrm{kV}$. With the largest capacitance used equal to $1 \mu \mathrm{F}$, the overlap voltage determined experimentally is $6 \mathrm{kV}$. Thus, the discharge mode may be considered as close to the limiting one which occurs under a constant voltage at electrodes.

\subsection{The Energy for Initiation of a High-Current Arc Discharge}

The question on the energy required to initiate a high-current arc is of importance from the standpoint of applications. The use of a discharge sliding over the conductor surface implies that rather high currents flow already during the incomplete stage of discharge, with the major portion of energy dissipating in the conductor bulk not shunted by the moving plasma formation. With the voltage close to the overlap voltage, almost the entire energy of the storage capacitor is released in the conductor, and such a system is of no practical significance.

In order to identify the conditions under which the portion of energy going into the discharge initiation is small compared to the initial energy stored in the capacitor, we integrated numerically the system of equations (3). We studied the efficiency of initiation as a function of initial strength of the electric field. The ratio of the energy remaining in the capacitor tank at the moment the discharge gap is overlapped by the leader to the initial stored energy was taken as a measure of efficiency $\varepsilon$. Let us clarify the notion of the storage-capacitor energy at the moment of overlapping. With Eqs. (3) solved formally, the voltage across the capacitor is zero at the moment the plasma front contacts the anode. This fact is a consequence of our assumption that the plasma column possesses an infinite condurtivity. Thus, formally, all the energy stored is dissipated within the conductor. However, from the physical standpoint, it is more correct to consider the moment when the resistance of the plasma column and that of the as yet nonoverlapped portion of the conductor become comparable as the moment when the discharge is shorted by the plasma. Therefore, in order to determine the initiation efficiency, we should consider the energy of the power-supply capacitor at the moment when the distance between the plasma front and the anode amounts to a fraction $\xi$ of the entire width of the discharge gap.

When determining the initiation efficiency $\varepsilon$, we assumed that $\xi=10^{-4}$. Similar calculations with $\xi$ varying from $10^{-2}$ to $10^{-6}$ demonstrated that the resulting spread in $\varepsilon$ is within $1 \%$.

Figure 6 a shows $\varepsilon$ as a function of reduced initial strength of the electric field $E_{a} / E_{c}$. A strong effect of characteristics of the discharge circuit and ambient gas enters via the dimensionless parameter $F=k r_{0} E_{c} C$. With increasing $F$, an increase in overlap voltage and a significant decrease in the efficiency of initiation are observed. As is evident from Fig. 6, the good points of the initiation method under consideration, namely, a low overlap voltage and low energy required for initiation, manifest themselves most clearly when the capacitance of the storage capacitor is large and the conductor has a high resistance per unit length.

A parameter of no less importance is the delay time $t_{d}$ between the arrival of a voltage pulse and the overlap of the discharge gap by plasma. Figure $6 \mathrm{~b}$ shows the ratio $\tau=\frac{t_{d}}{r_{0} C L}$ of the delay time $t_{d}$ to the time constant of the discharge circuit as a function of the reduced strength of the electric field. As is evident, the use of a system with large values of the parameter $F$ is preferable in this case as well. Thus, it is most appropriate to implement the method under consideration in high-energy systems with the capacitance of the storage capacitor being comparatively large. 

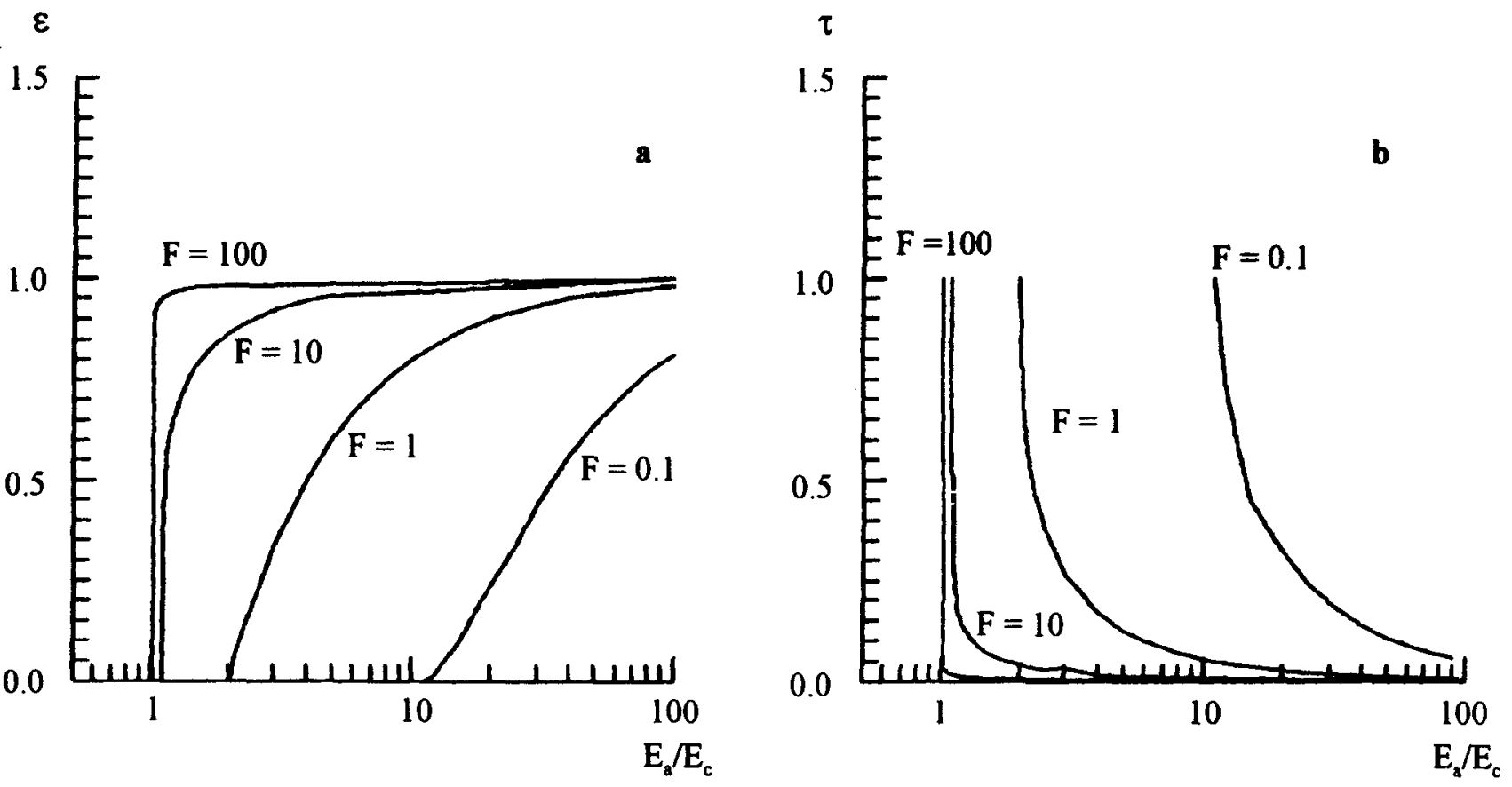

Fig. 6. Dependences of the efficiency of the discharge initiation $\varepsilon$ (a) and the delay time $\tau$ (b) on the reduced strength of the electric field $E_{a} / E_{c}$ for several values of the parameter $F$.

\subsection{The Mechanism of Formation of the Discharge That Slides over the Surface of a Conductor}

The experimentally observed high electric conductivity of moving plasma under atmospheric pressure of ambient gas indicates that we are dealing with an arc discharge. Thermal ionization is responsible for the generation of charged particles in the plasma of such a discharge. In contrast, propagation of the plasma front may be due to various mechanisms of the transfer of the energy and ionization state from the discharge plasma to adjacent layers of unheated gas; these mechanisms include thermal conduction, shock wave, radiant heat exchange, diffusion of electrons, and resonance emission. Let us consider in more detail the conditions in which the strength of the applied electric field only slightly exceeds the threshold value required for initiation of the mode of propagating discharge. In that case, the motion velocity of the plasma front is low, and, apparently, there is an analogy here with the mechanism of the discharge-front propagation in the generators of equilibrium low-temperature plasma, i.e., plasmatrons. In such discharges, the transfer of the state ionization is provided by the most universal mechanism, i.e., by the thermal-conduction transport of heat to the region of unheated gas with subsequent thermal ionization of this gas.

In order to develop a quantitative theory of the process, we have to solve a complete system of gas dynamics equations with allowance for thermal conduction and Joule's energy release in the bulk of the plasma under the effect of current. However, taking into account that the flow is subsonic, we consider the case of constant pressure equal to the pressure of the ambient gas. A simultaneous solution of gasdynamic and electric-field equations is a complicated problem even in this case.

We would like the model under consideration to account for the most important qualitative characteristics of the discharge development; with this in mind, we consider the problem of plasma-front propagation in the idealized one-dimensional case. Assuming that the front motion is steady-state with velocity $u$, we write the 
equation of energy balance as

$$
u \rho \frac{c_{p}}{\lambda_{p}} \frac{d \theta}{d x}=\frac{d^{2} \theta}{d x^{2}}+Q-L
$$

with the boundary conditions

$$
\theta(x \rightarrow-\infty), \quad \frac{d \theta}{d x}(x \rightarrow+\infty)=0,
$$

where the coordinate $x$ is measured from the region of unheated gas ahead of the front in the direction toward the plasma; $\lambda_{p}$ and $c_{p}$ are the thermal conductivity and specific heat of the gas under constant pressure; $\theta=\int_{T_{0}}^{T} \lambda_{p} d T$ is the thermal potential at temperature $T ; T_{0}$ is room temperature; $Q$ is the intensity of Joule heating per unit volume; $L$ is thermal losses from the unit volume of plasma; and $\rho$ is the density of the gas, provided that the thermal flux $\theta_{0}$ is given by $Q\left(\theta_{c}\right)=L\left(\theta_{c}\right)$.

In order to determine the function of heat losses $L$, we invoked the experimental fact that a moving plasma channel is of the shape of a semicylinder with diameter equal to the face of the ferrite sample along which the motion occurs. Therefore, in writing the equations of energy balance (8), we replaced the transverse component of the heat-flux divergence with the expression

$$
L=\frac{A \theta}{r^{2}}
$$

where $r$ is the radius of a semicylinder and $A$ is a dimensionless coefficient. Such a formulation of the problem is similar to that in the theory of the thermal-conduction regime of the optical-discharge propagation, which was developed in [8]. The numerical coefficient was determined from a solution of the two-dimensional equation of thermal conduction $\Delta \theta+L=0$. The problem was solved at the semicircle of radius $r$ with zero conditions at the boundary and $L=$ const. As a result, we obtained $A=10.24$.

In order to determine the rate of energy release $Q$, we consider the case of sufficiently small cross sections for both the plasma channel and the conductor over whose surface the discharge develops. Then, ignoring the nonuniformity of the current distribution over the conductor and plasma cross sections, we represent the equivalent electric circuit of the conductor-plasma channel system as two resistances connected in parallel. The expression for the rate of energy release in the plasma column then takes the form

$$
Q=\frac{\sigma_{p} J^{2}}{\left(\sigma_{p} s+\sigma_{0} S\right)^{2}}
$$

where $J$ is the total current through the plasma and conductor; $\sigma_{p}$ and $\sigma_{0}$ are the conductivity, respectively, of the plasma and the conductor; and $s=\pi r^{2} / 2$ and $S$ are the cross-sectional areas of the plasma channel and the conductor.

The problem of determining the propagation velocity of the plasma front $u$ reduces to the problem of finding the eigenvalues of Eq. (8) complemented with relevant boundary conditions. We numerically calculated the eigenvalues with the use of expression (9) for the heat-loss function and expression (10) for the heat-release rate. Thermodynamic functions and transport coefficients for gases at atmospheric pressure were taken from [9]. In numerically solving Eq. (8), we took into consideration the nonlinear dependence of the ferrite-slab conductivity $\sigma_{0}$ on the applied electric field $E$; based on our experimental data, we approximated this dependence as

$$
\sigma_{0}= \begin{cases}0.509 \ln E-3.294, & \text { for } E>655 \mathrm{~V} / \mathrm{cm} \\ 4.35 \cdot 10^{-3}, & \text { for } E \leq 665 \mathrm{~V} / \mathrm{cm}\end{cases}
$$


where we used the units of $\mathrm{V} / \mathrm{cm}$ for the field $E$ and $\Omega^{-1} \mathrm{~cm}^{-1}$ for the conductivity $\sigma_{0}$. Since the model is based on the assumption that the flow is subsonic, the calculation was continued until the plasma front reached the sonic velocity of propagation.

The results of comparison of calculated and experimental data on the propagation velocity of the plasma front in the air and argon ambients at atmospheric pressure are shown in Fig. 5, where the solid and dashed lines correspond to the results of calculations. It is evident that experimental and theoretical dependences agree with each other both qualitatively and quantitatively. The difference in the rates of increase of propagation velocity for air and argon is attributed, on the basis of the results obtained, to the fact that, notwithstanding practically the same temperature dependence of electrical conductivity for both gases, the thermal conductivity of argon is lower than that of air by several orders of magnitude, which results in lower heat losses from the plasma column.

Thus, we may posit that the main mechanism responsible for discharge propagation over the surface of a conductor in the vicinity of the threshold is related to thermal conduction. However, as the initial field strength and total current increase, the temperature and electrical conduction of the plasma rise. The effect of enhancement of the electric-field strength ahead of the plasma front, as we observed experimentally, becomes more and more pronounced. In this case, a change of the discharge-propagation mechanism can occur. The electrons in plasma diffuse into the region of gas preliminarily heated and subjected to a high electric field and are able there to ionize the gas. The propagation regime thus developed is similar to the one in the case of corona discharge, but features an efficient transfer of excess negative charge to the conductor.

The model we developed does not provide insight into the different behavior of the anode- and cathodedirected plasma channels. It follows from the model that the velocities of both channels are bound to be the same. However, under the conditions of our experiments, we invariably observed the preferential development of the anode-directed channel, whereas anode- and cathode-directed channels moving with the same velocity were reported in [3].

This question can be settled qualitatively by considering the conditions of closing the current lines in the plasma-conductor surface system. In the case of a plasma front moving to the electrode of negative polarity, the conductor surface acts as a cathode. This means that an electron current should flow from the conductor to the plasma. With low temperature of the surface, i.e., at low electric fields applied and for low conductivity of the conductor material, electrons can be generated owing to $\gamma$ processes at the surface, the efficiency of which is low. Therefore, in order to provide the conditions for plasma current, we have to heat the surface to temperatures sufficiently high for thermoionic emission to become of importance. Thus, the propagation velocity of the cathode-directed plasma channel is governed by the velocity of motion of the temperature wave, which is related to the thermal diffusivity of the conductor material.

The role of the conductor surface in the process of the anode-directed plasma channel is substantially different. The conductor serves as an anode in reference to the plasma, and, therefore, its function is a passive collection of electrons from the plasma. Thus, the condition of the conductor surface should not, in general, affect the propagation velocity of the plasma front. It follows from the above that a preferential development of the anode-directed leader should occur in the range of low currents; in that case, the leader velocity can be estimated on the basis of our model. With increasing current, the heating of the surface to high temperature takes less time and is no longer a factor impeding the development of the cathode-directed leader. The velocity of the latter becomes comparable to the propagation velocity of the front of the anodedirected leader, and the discharge model based on thermal conduction turns out to be valid in this case as well.

A more thorough study of the interaction of discharge plasma with the conductor surface is a separate problem, related to a consideration of the mechanisms of formation of the regions of anodic and cathodic potential drops in the vicinity of contacts between plasma and conductor; these issues were outside the scope of this work. 


\section{Summary and Conclusion}

In this work, we studied a poorly understood form of gas discharge, namely, the arc discharge sliding over the surface of a conductor. Experimental studies were performed over a wide range of pressure of different gases and energy supplied to the discharge. Along with the theoretical analysis performed, these studies made it possible to identify the mechanism of discharge formation. In particular, we succeeded in combining widely differing concepts of the origin of such discharges $[2,3]$; these concepts are shown to be related to limiting cases of the realization of these discharges.

We experimentally determined the dependences of the discharge-front propagation velocity and the breakdown (overlap) voltage on the pressure and type of ambient gas and on the parameters of the discharge circuit. Our experiments verified the fact postulated in [2] that the electric-field strength is appreciably enhanced ahead of the plasma front owing to the high conductivity of the plasma channel. As a result of comparison of experimental data with the model we developed, it was established that the mechanism responsible for the formation of a sliding arc discharge in the vicinity of the threshold is heating of the gas ahead of the plasma front, which is due to thermal conduction and is followed by thermal ionzation.

Based on the data obtained, a general pattern of the regimes in the discharge formation appears as follows: In the threshold region, which features a low electrical conductivity of the discharge plasma, the thermalconduction-related mechanism is of crucial importance in stimulating the plasma-front propagation, which is consistent with the viewpoint in [3]. An increase in the strength of the applied electric field results in a rise in temperature, an increase in electrical conductivity of the plasma, and enhancement of the field ahead of the plasma front. As this takes place, in accordance with [2], the process of impact ionization in the enhanced electric field may become the main mechanism of discharge.

\section{References}

1. S. Hesketh, "The propagation of arcs over a water surface," Proceedings of the VIIIth Intern. Conference on Phenomena in Ionized Gases, Vienna (1987), p. 255.

2. B. Kh. Brodskaya, Khim. Vys. Energ., 16, 458 (1982).

3. E. A. Azizov, N. A. Akhmerov, G. G. Gladush, and I. P. Shed'ko, Teplofiz. Vys. Temp., 22, 655 (1984).

4. S. V. Mit'ko, V. N. Ochkin, A. V. Paramonov, and A. P. Shirokikh, "A high-brightness gas discharge over the conductor surface," Proceedings of the VIII Soviet Conference on Physics of Low-Temperature Plasma [in Russian], Minsk (1991), Part 1, p. 97.

5. S. V. Mit'ko, A. V. Paramonov, and A. P. Shirokikh, "Comparison of emission characteristics of the highpower electric discharges initiated by ferrite and exploding wire," Proceedings of the II Soviet Symposium on Radiation Plasmodynamics RPD-91 [in Russian], Moscow State Technical University, Moscow (1991), Part 1, p. 35.

6. S. V. Mitko and V. N. Ochkin, J. Russ. Laser Res., 17, 259 (1996).

7. S. V. Mitko, F. A. van Door, V. N. Ochkin, and W. J. Witteman, "Optical pumping and ferrite flash discharges," Proceedings of the NATO Res. Workshop ARW 950443 on Gas Lasers - Recent Developments and Future Prospects, Kluwer, Netherlands (1996), p. 185.

8. Yu. P. Raizer, "Optical discharges," Usp. Fiz. Nauk, 132, 549 (1980).

9. Yu. P. Raizer, Physics of Gas Discharge [in Russian], Nauka, Moscow (1987). 